\title{
Atmospheric Dispersion Simulations for the Assessment of Radiological Dose to the Public
}

\author{
-Reassessment of the Atmospheric Concentration \\ Distribution of Radioactive Materials in the Immediate \\ Aftermath of the Accident at the Fukushima Daiichi \\ Nuclear Power Plant-
}

\author{
Japan Atomic Energy Agency, Haruyasu Nagai
}

\begin{abstract}
Radioactive materials were released into the environment due to the accident that occurred at the Fukushima Daiichi Nuclear Power Plant, which is operated by the Tokyo Electric Power Company. This release immediately led to the performance of an internal exposure dose assessment of iodine and other nuclides with a short halflife. To determine the necessary dose estimation, the spatial-temporal distribution of the atmospheric concentration of radioactive materials was reassessed by performing dispersion simulations with ${ }^{131} \mathrm{I},{ }^{133} \mathrm{I},{ }^{132} \mathrm{Te}$, and ${ }^{137} \mathrm{Cs}$ with due consideration given to their contribution to the internal exposure doses. A database of the spatial-temporal distribution of the concentration was developed based on the results obtained from the calculations performed for each defined time at a horizontal interval of $3 \mathrm{~km}$ near the ground surface.
\end{abstract}

\section{Introduction}

Radioactive materials were released into the environment due to the accident that occurred at the Fukushima Daiichi Nuclear Power Plant, which is operated by the Tokyo Electric Power Company (TEPCO). The exposure doses must be tracked as they serve as basic data for assessing the health risks faced by local residents of Fukushima Prefecture and its surrounding areas. At present, it is difficult to perform an assessment of the internal exposure doses for iodine and other nuclides with a short half-life based on measurements during the initial post-accident phase. A realistic approach to determining the necessary estimation is to combine a behavior pattern and a chronological atmospheric concentration map of the radioactive materials from an atmospheric dispersion simulation. The Japan Atomic Energy Agency (JAEA) is conducting a detailed analysis of the transport of the nuclear materials released into the environment due to the accident ${ }^{1-4)}$ by employing the System for Prediction of

DOI : 10.15669/fukushimainsights.Vol.2.37

(C) 2021 Atomic Energy Society of Japan. All rights reserved.

Originally published in Journal of the Atomic Energy Society of Japan (ISSN 1882-2606), Vol. 55, No. 12, p. 712-717 (2013)

in Japanese. (Japanese version accepted: September 16, 2013) 
Environmental Emergency Dose Information (SPEEDI), which was developed for responding to nuclear emergencies, and the worldwide version of SPEEDI (WSPEEDI) ${ }^{5}$.

This commentary explains how WSPEEDI was employed in the detailed atmospheric dispersion analysis of the radioactive materials released into the environment due to the Fukushima Accident. The analysis performed using atmospheric dispersion simulations was intended to develop a spatial-temporal distribution database of the atmospheric concentration of radioactive materials, which is needed for the dose estimation.

\section{Analysis Method}

\section{Source Conditions}

In preparation for atmospheric dispersion simulations, temporal changes in the release rates for iodine and other nuclides with a short half-life, the release heights, and other source conditions (source term) were investigated and compiled based on open information and publications. These factors were organized as input conditions for performing the simulations. To validate the results produced by these atmospheric dispersion simulations, the source conditions for conducting simulations of ${ }^{137} \mathrm{Cs}$ and other nuclides with a long half-life, for which a relatively abundant amount of local measurement data is available, were also prepared. More specifically, the JAEA has published papers in scientific journals to present the estimated sources of ${ }^{131} \mathrm{I}$ and ${ }^{137} \mathrm{Cs}$ based on a combined analysis conducted using both atmospheric dispersion simulations and monitoring data ${ }^{1-4)}$. These estimated sources are the only estimation results that accommodate changes to the settings for the release rate during the target period and enable the reproduction of environmental monitoring results. These results were then compared with other estimation results as well as the results of an analysis of the reactor interiors to closely examine the source conditions and prepare the input conditions for simulations.

\section{Atmospheric Dispersion Analysis}

The atmospheric dispersion simulations were conducted by reproducing the meteorological field that existed during the accident in a three-dimensional meteorological model and subsequently calculating the transport, dispersion, and deposition of radioactive materials based on the abovementioned source conditions. The analysis was carried out using the meteorological model MM5 and the atmospheric dispersion model GEARN of WSPEEDI ${ }^{5)}$, which was developed by the JAEA.

The meteorological model MM5 calculates the three components of wind speed, turbulence quantities, and precipitation as well as other meteorological factors that are needed to calculate the atmospheric dispersion based on numerical forecast data provided by the Japan Meteorological Agency and other initial conditions. This model numerically solves the equations for the three momentum components that take into consideration the spherical effect of the Earth and topographical influence as well as factors such as thermal energy, the water vapor quantity, and the cloud water content (liquid and solid). The atmospheric dispersion model GEARN derives the atmospheric concentration of radioactive materials and the quantity deposited on the ground surface by calculating the transport and dispersion of these materials based on the source conditions and meteorological factors obtained using the meteorological field calculation. This model also calculates the air dose rate based on the atmospheric 
concentration of radioactive materials as well as air dose rate affected from the radionuclides deposited on the ground surface. Given the need to produce a precise evaluation of the radiation impact caused by a localized high-concentration distribution near the source, GEARN employs a Lagrangian particle dispersion model to take into consideration attenuation by the radioactive decay of the respective released nuclides. On the ground surface, both dry deposition due to turbulent flow and wet deposition due to precipitation are taken into consideration. The calculation of the wet deposition takes into account the three-dimensional distribution of precipitation and cloud cover derived from the three-dimensional meteorological model. The precision of these models has been verified by the Chernobyl Accident analysis, and real-time predictions and performance assessment of post-test analysis conducted in the European Tracer Experiment.

The exposure dose assessment targets onshore areas within $250 \mathrm{~km}$ of the source. Accordingly, the atmospheric concentration and other factors considered in atmospheric dispersion simulations were calculated over an area extending $690 \mathrm{~km}$ east-west and $960 \mathrm{~km}$ north-south with a horizontal spatial resolution of $3 \mathrm{~km}$. The behavior of the radioactive materials released into the atmosphere within the target period from 0:00 on March 11 to 24:00 on April 30, 2011, was continuously calculated.

\section{Analysis Results}

\section{Source Conditions}

Calculations were performed with ${ }^{131} \mathrm{I},{ }^{133} \mathrm{I},{ }^{132} \mathrm{Te}$, and ${ }^{137} \mathrm{Cs}$, taking into consideration their contribution to the internal exposure doses based on the estimated source term. The amount of ${ }^{137} \mathrm{Cs}$ was also used in a comparison with measured data on the deposition quantity. The time variations of the release rate were used by those estimated by the JAEA for ${ }^{131} \mathrm{I}$ and ${ }^{137} \mathrm{Cs}$ (Figure 1) ${ }^{4}$. For other nuclides with a short half-life $\left({ }^{133} \mathrm{I}\right.$ and $\left.{ }^{132} \mathrm{Te}\right)$ the radioactivity ratio to ${ }^{131} \mathrm{I}$ and ${ }^{137} \mathrm{Cs}$ were estimated based on the limited environmental monitoring data, decay coefficients, and estimated inventory.

Figure 2 compares the radioactivity ratio in the inventory for each time of release with the ratio of radioactivity according to data from the atmospheric concentration measurements. Based on nuclide characteristics, the condition of ${ }^{132} \mathrm{Te}$ in the atmosphere is presumably similar to that of ${ }^{131} \mathrm{I}$. However, the ratio of ${ }^{132} \mathrm{Te}$ to ${ }^{131} \mathrm{I}$ in a dust sample demonstrates a low

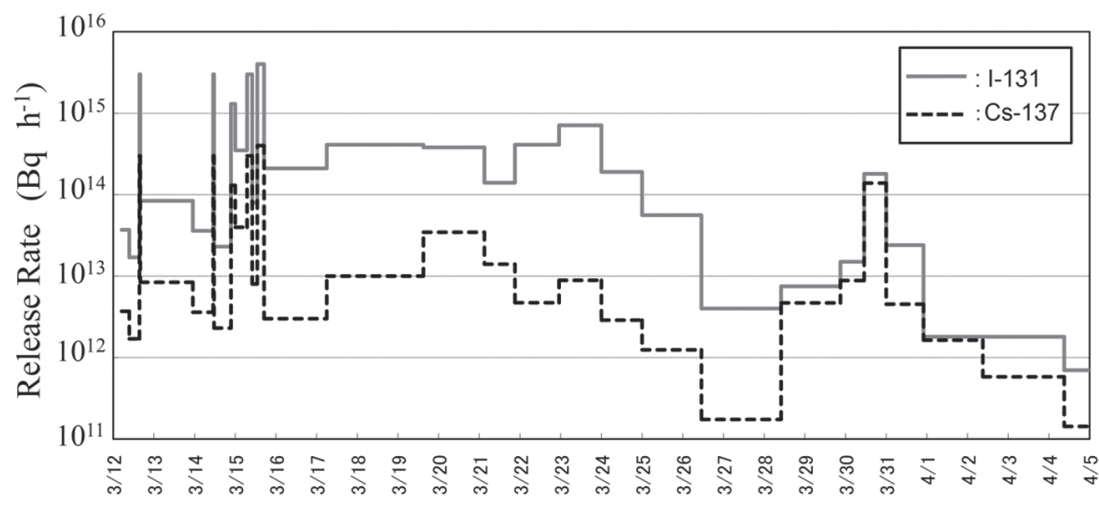

Figure 1 Time variation of release rates for ${ }^{131} \mathrm{I}$ (solid line) and ${ }^{137} \mathrm{Cs}$ (dashed line) 

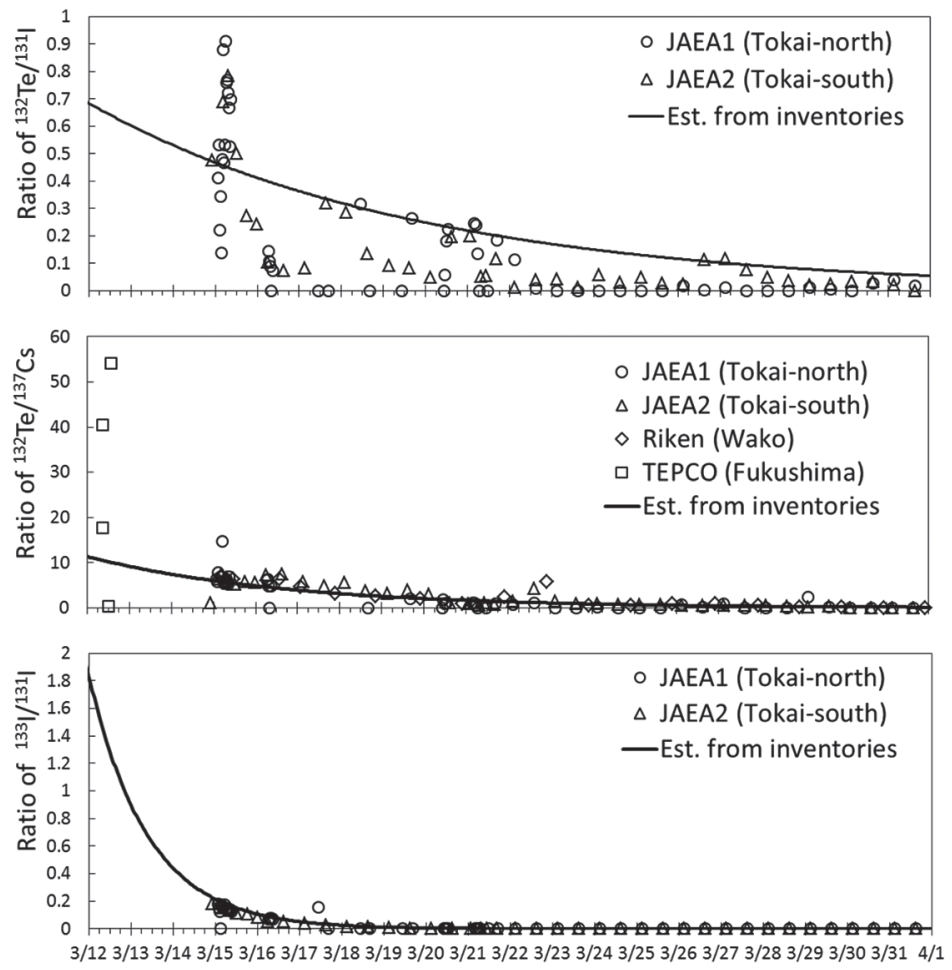

Figure 2 Comparison of time variation of radioactivity ratio of ${ }^{133} \mathrm{I}$ and ${ }^{132} \mathrm{Te}$ to ${ }^{131} \mathrm{I}$ or ${ }^{137} \mathrm{Cs}$

( $\bigcirc, \triangle, \diamond$, and $\square$ indicate the radioactivity ratios from the dust sampling, while solid lines represent the radioactivity ratios estimated from the inventory)

correlation with the ratio of radioactivity in the inventory during the release. Meanwhile, strong correlations can be observed with respect to the radioactivity ratio of ${ }^{132} \mathrm{Te}$ to ${ }^{137} \mathrm{Cs}$ from the dust sample and those from the inventory during the release. Adjusting the radioactivity ratio for the initial inventory to ${ }^{132} \mathrm{Te} /{ }^{137} \mathrm{Cs}=20$ produced a satisfactory reproduction of the radioactivity ratio in the dust sample with the radioactivity ratio in the inventory during the release. The behavior of ${ }^{133} \mathrm{I}$ during its release and atmospheric dispersion is assumed to be the same as that of ${ }^{131} \mathrm{I}$. In the dust sample and inventory, changes in the radioactivity ratio almost overlap each other. Accordingly, the radioactivity ratio of ${ }^{133} \mathrm{I}$ to ${ }^{131} \mathrm{I}$ can probably be adopted directly from the ratio in the inventory during the release.

The abovementioned changes in the release rate for each nuclide were assigned as the basic source conditions to investigate their uncertainty ranges and the resultant impact on the calculation of the concentration. The source term estimation result produced by the JAEA to assign temporal changes in the release rate for ${ }^{131} \mathrm{I}$ and ${ }^{137} \mathrm{Cs}$ has already been verified through a comparison with some reproductive tests that were conducted using environmental monitoring data based on a simulation of the atmospheric dispersion and an estimation of the release rates by Japanese and overseas research institutes. A comparison with the estimates ${ }^{6}$ produced by Hirao et al. from Nagoya University demonstrated almost the same changes over time. Hirao et al. have also evaluated the uncertainty associated with the estimated release rates and concluded that the estimated values have a range with a factor of 3 (ranging from $1 / 3$ to 3 times). Similar to the JAEA, Hirao et al. adopted a method of deriving a release rate that reproduces the environmental monitoring data by means of an atmospheric dispersion simulation. As they commonly employ similar atmospheric dispersion models, the estimates produced by the JAEA were assumed to have a range with a factor of approximately 3 to assess 
the impact of uncertainty associated with the source information.

\section{Atmospheric Dispersion Analysis}

Based on the abovementioned source conditions, the predicted accuracy was evaluated by comparing simulations produced by using WSPEEDI and the actual measurements for the air dose rates, the airborne nuclide concentration, and the distribution of the ${ }^{137} \mathrm{Cs}$ deposition.

To address the process involved in the dispersion of released radioactive materials in the atmosphere and the contamination of the ground surface, the JAEA held an open workshop on March 6, 2012 entitled "Reassessment of the environmental release and dispersion process associated with the accident at the Fukushima Daiichi Nuclear Power Plant." Based on the discussions with participants from other organizations, the key plume movements and the deposition process that formed the deposition distribution of ${ }^{137} \mathrm{Cs}$ as observed by aircraft monitoring were summarized as follows.

(1) March 12: The plume passed Minamisoma in the direction of the sea and then passed through the environs of the Onagawa Nuclear Power Plant, causing dry deposition in these areas.

(2) March 15: The plume released before dawn travelled southward along the coast, causing dry deposition in the area located between Iwaki and northern Ibaraki.

(3) March 15-16: The same plume moved inland from Ibaraki on the Kanto Plain, causing wet deposition in Gunma Prefecture and Tochigi Prefecture. The plume released before noon traveled southwest and west, causing wet deposition in Nakadori, Fukushima Prefecture. A highly concentrated plume released in the afternoon was carried northwest from the nuclear power plant, causing wet deposition to form a highly contaminated area.

(4) March 20: The plume moved northeast after flowing northwest, causing wet deposition while passing through the area between northern Miyagi and southern Iwate.

(5) March 21: The plume traveled southward over the sea, causing wet deposition while passing through the area between southern Ibaraki and northwestern Chiba to form a hot spot.

Based on these results, an evaluation was conducted to assess the reproducibility of the plume movements and the associated deposition.

As an example, Figure 3 compares the time variations of the calculated and measured air-absorbed dose rates during Event (2) at the Fukushima Daini Nuclear Power Plant, Iwaki, Kitaibaraki, and Mito. This comparison presents not only computational grids that correspond to the observation points, but also grids within $3 \mathrm{~km}$ or $9 \mathrm{~km}$ of the observation points. The reproducibility of the measurements is significantly compromised if the course taken by the plume deviates even slightly in a comparison with spatially discrete measurement data. Therefore, the comparison in Figure 3 is intended to gauge the extent of the misalignment in such cases. Changes at each of the points in eastern Japan were almost reproduced, but there were two- to three-hour misalignments in the timing of the plumes as they passed over some points depending on the periods and areas. In some cases, the plumes passed a few meshes apart. An exposure dose assessment based on an atmospheric dispersion simulation needs to take into consideration the uncertainty associated with such spatial-temporal misalignments of the passing plumes.

With respect to the time histories of airborne nuclide concentration, Figure $\mathbf{4}$ compares the calculated and measured atmospheric concentration of ${ }^{131} \mathrm{I}$ when plumes passed over Tokai-mura (JAEA) from March 14 to 17 and 20 to 21. Although the reproducibility of the time variations was similar, some cases of quantitative underestimation imply that the 

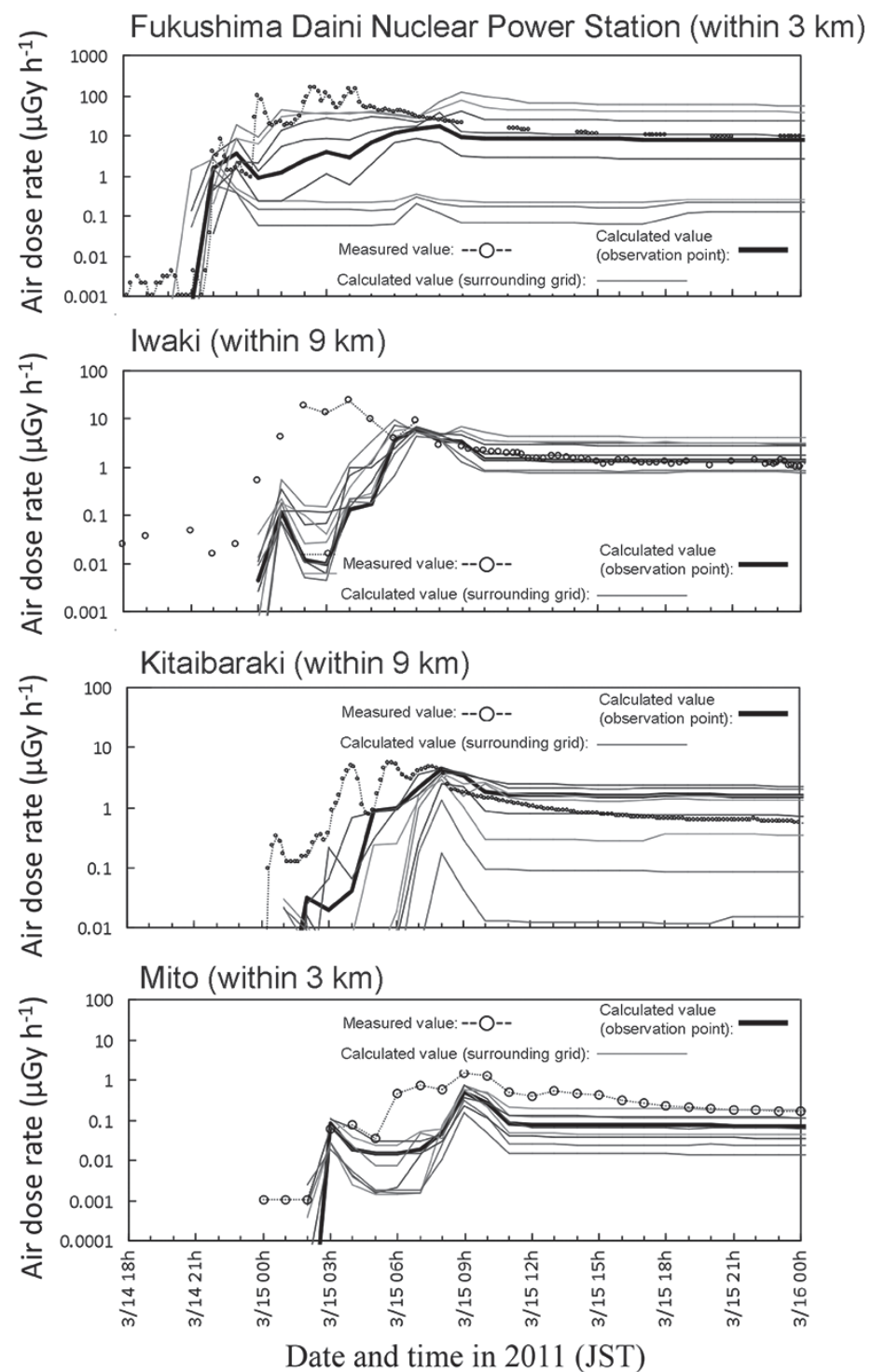

Figure 3 Comparison of measured and calculated air-absorbed dose rates when a plume passed over the Fukushima Daini Nuclear Power Station (Plant), Iwaki, Kitaibaraki, and Mito on March 15 (The calculated values correspond to the grids covering the observation points and the grids within $3 \mathrm{~km}$ or $9 \mathrm{~km}$ of these points)

uncertainty associated with the source conditions had an impact.

Compared with the distribution measured by aircraft monitoring, WSPEEDI overestimated the deposition of ${ }^{137} \mathrm{Cs}$ in Miyagi Prefecture and underestimated it in Gunma and Tochigi Prefectures. This is probably attributable to the application of the uniform precipitation scavenging rate without taking into consideration vertical variations in the conditions for the wet deposition process. The reproducibility improved when the following factors were taken into consideration: the actual weather that prevailed during the accident; differences in the scavenging rates in the ice and liquid phases as well as inside and under the clouds; and the impact from fog deposition. The impact assessment is being conducted through an analysis that 

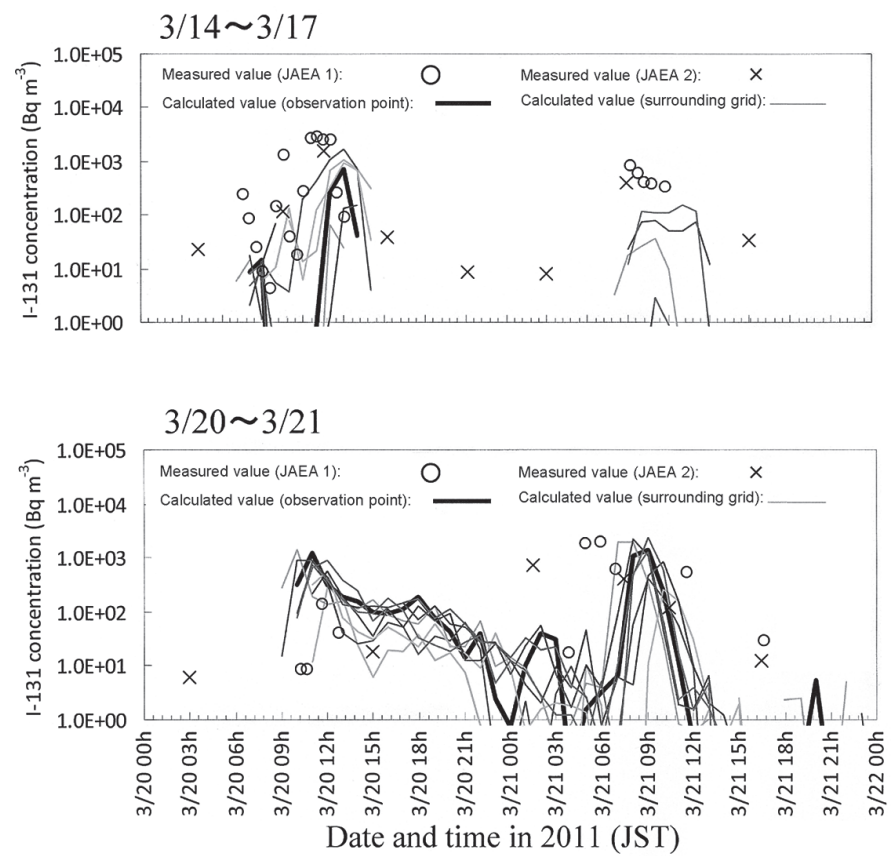

Figure 4 Comparison of the measured and calculated atmospheric concentration of ${ }^{131} \mathrm{I}$ when the plumes passed over Tokai-mura (two observation points at the JAEA) from March 14 to 17 and 20 to 21 (The calculated values correspond to the grids covering the observation points and the grids within $18 \mathrm{~km}$ from there)

takes into consideration differences in the dry deposition velocity and the scavenging rate depending on the forms of the airborne nuclides.

The uncertainty associated with the source conditions and the atmospheric dispersion simulations must be addressed to perform more precise exposure dose assessments. The source conditions are inversely estimated from the measured radioactive nuclide concentration through atmospheric dispersion simulations. Given this, the source conditions are influenced by the uncertainty associated with the model calculations. Because the simulations are based on spatially and temporally discrete measurement data, uncertainty is further compounded by the use of an interpolation method and the assumptions made in calculating the dispersion, such as the release height and duration. To address this uncertainty, it is necessary to enrich the measurement data and conduct an analysis again using an improved model. However, improvements to the model crucially require a comparison of the calculations based on accurate source conditions and measurement data. Going forward, we need to establish an analysis method that meets these almost contradictory requirements.

\section{Impact of Modifications to the Deposition Process}

The possibility of improving reproducibility of the distribution of the ${ }^{137} \mathrm{Cs}$ deposition by aircraft monitoring was demonstrated after modifications had been made to the deposition process in the dispersion calculation. Accordingly, the degree of its impact was investigated on the calculation of the atmospheric nuclide concentration near the ground used for an internal exposure dose assessment. More specifically, the integrated atmospheric concentrations near the ground surface were compared to evaluate the maximum extent of the impact by 
examining the following: the difference between cases with completely gaseous radioactive nuclides and cases with completely particulate nuclides; the difference observed with onetenth of the scavenging coefficient in the ice phase; and the difference between cases with the maximum and minimum scavenging coefficients for particulate nuclides.

The transition of completely gaseous radioactive nuclides into completely particulate nuclides caused a significant increase in the deposition quantity and the integrated atmospheric concentration near the ground surface. Given that radioactive nuclides in the atmosphere are removed due to deposition, the increased removal quantity and the increased atmospheric concentration seem contradictory. Nonetheless, this result is valid. It can be explained by the difference in the deposition processes and the three-dimensional distribution of the removal quantity. With the parameters applied on this occasion, the dry deposition velocity is six times higher with completely gaseous radioactive nuclides than it is with completely particulate nuclides. However, the precipitation scavenging coefficient is lower with completely gaseous radioactive nuclides than that with completely particulate nuclides. Hence, when completely gaseous radioactive nuclides are replaced by completely particulate nuclides, they experience significantly less dry deposition and an increased level of wet deposition. In other words, wet deposition results in a large deposition quantity as clouds and precipitation removes radioactive nuclides from the atmosphere at high altitudes. In contrast, dry deposition removes atmospheric nuclides near the ground surface, causing deposition. However, this is because the reduction in the concentration there becomes larger, although the deposition quantity may be small. Any change in the proportion of gaseous and particulate radioactive iodine causes a considerable change in the atmospheric concentration near the ground surface, thereby increasing the impact on an internal exposure dose assessment.

Modifying the scavenging coefficient in the ice phase to 1/10 improved the overestimation of the ${ }^{137} \mathrm{Cs}$ deposition distribution in Miyagi Prefecture. However, the integrated atmospheric concentration near the ground surface barely changed. Since any change in the scavenging process in the ice phase affects only the cool parts of a plume at high altitude, the atmospheric concentration near the ground surface remained almost unchanged. For this reason, the enhanced reproducibility of the ${ }^{137} \mathrm{Cs}$ deposition distribution with a modified scavenging coefficient in the ice phase probably has little impact on an internal exposure dose assessment.

As you would normally expect, any difference between the maximum and minimum scavenging coefficients for particulate nuclides caused the integrated atmospheric concentration near the ground surface to increase as the deposition quantity decreased. In particular, the deposition quantity and the integrated atmospheric concentration near the ground surface changed significantly in areas with a predominant wet deposition when plumes passed during (3) March 15 to 16 and (5) March 21. Careful attention must be given to this point during an internal exposure dose assessment.

\section{Time-Series Atmospheric Concentration Map of Radioactive Materials}

A time-series atmospheric concentration map of radioactive materials was prepared by outputting the distribution of the atmospheric concentration near the ground surface and the ground surface deposition of each nuclide as calculated in the atmospheric dispersion simulation produced using WSPEEDI. The output was performed at a horizontal interval of $3 \mathrm{~km}$ in both the east-west and north-south directions every hour on the hour (average value in one hour). The calculation method was adjusted to output a database that facilitates the later assignment of source conditions after an atmospheric dispersion simulation has been performed using unit release conditions. In addition, databases were created for each case using the 
assigned parameters for gaseous and particulate materials based on analysis results that reflect the considerations taken with respect to the deposition process in the dispersion simulation described earlier. These cases could be combined to create dispersion analysis results for any proportions of gaseous and particulate materials.

To create each database with reference to the assigned changes in the release rate over time in Figure 1, a calculation is performed by assigning the unit release rate $\left(1 \mathrm{~Bq} \mathrm{~h}^{-1}\right)$ of each nuclide for one segment (e.g., 30 segments, with the first segment lasting from 5 am to 9:30 am JST on March 12) at a constant release rate. Regardless of the availability of the calculated values for the atmospheric concentration and deposition, the followings are output at an interval of one hour throughout the target period: the calculated concentration, the deposition quantity, and the air dose rate of each nuclide. This calculation is performed for all $30 \mathrm{seg}$ ments at a constant release rate. The outputs are stored as a database in a directory format

$\mathrm{I}-131$

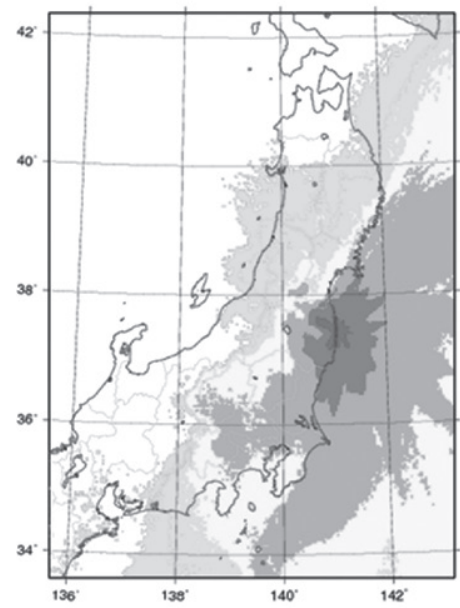

$\mathrm{I}-133$

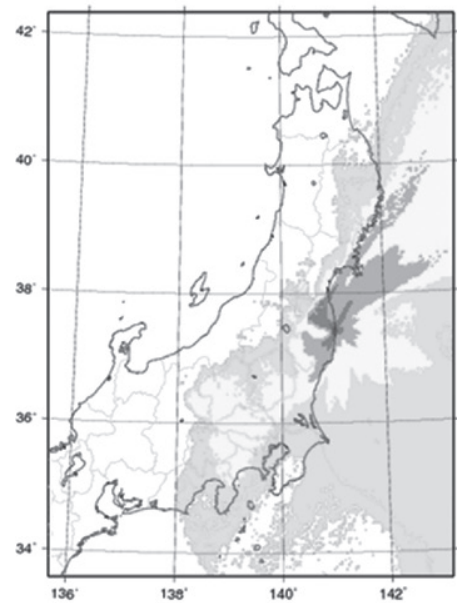

Cs-137

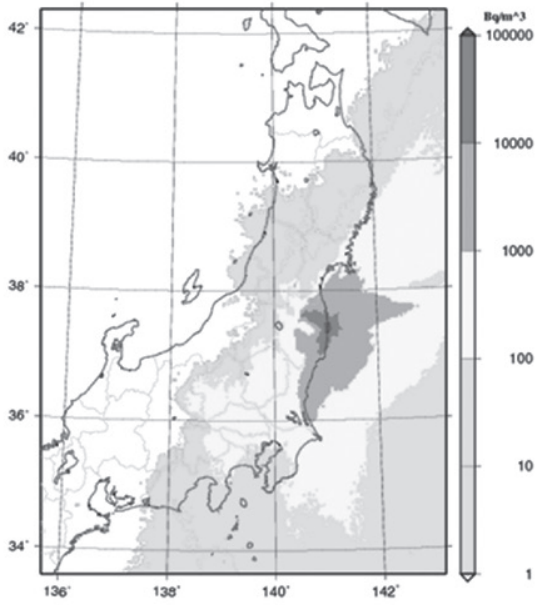

Te-132

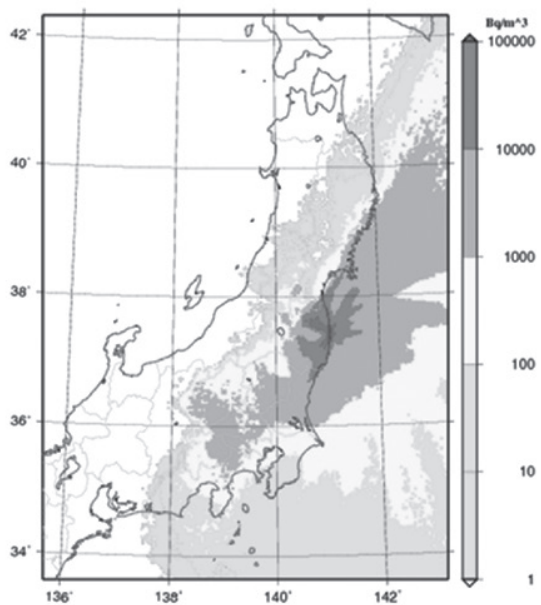

Figure 5 Integrated atmospheric concentration of ${ }^{131} \mathrm{I},{ }^{133} \mathrm{I},{ }^{137} \mathrm{Cs}$, and ${ }^{132} \mathrm{Te}$ near the ground surface during the assessment period from 00:00 on March 11 to 24:00 on April 30, 2011 
associated with each segment. To produce the calculation results for the source conditions assigned by the database, the release rate for each nuclide assigned to each segment at a constant release rate is multiplied by the outputs for the concentration, the deposition quantity, and the air dose rate of each nuclide for the entire period stored in the associated directory. Subsequently, outputs that correspond to the same time are combined. This method demonstrated an almost identical output as that for the dispersion simulations performed by assigning source conditions in the beginning.

This calculation method was employed to create databases for nine cases using different model parameters for gaseous and particulate materials. The results of the dispersion analysis can be produced for any proportion of gaseous and particular materials by combining the outputs from each case prepared by adjusting the source conditions.

Figure 5 shows the distribution of the integrated atmospheric concentration of each nuclide near the ground surface during the target period as prepared by applying the basic source conditions to these databases. The integrated concentration distribution varies according to different temporal changes in the release rate of each nuclide. In particular the distributions for ${ }^{133} \mathrm{I}$ and ${ }^{132} \mathrm{Te}$ with a short half-life indicate a large release rate in the immediate aftermath of the accident when the plume was being carried northeast.

\section{Conclusions}

The Fukushima Daiichi Nuclear Accident immediately led to the performance of an internal exposure dose assessment of iodine and other nuclides with a short half-life from among the various radioactive materials released into the environment. To determine the necessary internal dose estimation, database for the spatial-temporal distribution of the atmospheric concentration of radioactive materials was constructed by performing dispersion simulations. The analysis results include uncertainty associated with the source conditions and the model calculation, but they can be applied to produce rough estimates of the dose. Nonetheless, they are not yet precise enough. The level of precision must be further enhanced by improving the model and scrutinizing the source conditions.

The analysis presented in this commentary was subcontracted by the National Institute of Radiological Sciences to develop time-series maps of the atmospheric concentration of radioactive materials through the performance of atmospheric dispersion simulations. This was done as part of an internal exposure dose assessment of iodine and other nuclides with a short half-life in the immediate aftermath of the nuclear accident under a project led by the Ministry of the Environment to assess the impact of the Fukushima nuclear emergency in FY2012.

\section{References}

1) M. Chino, H. Nakayama, H. Nagai, H. Terada, G. Katata, and H. Yamazawa: J. Nucl. Sci. Technol., 48, 1129-1134 (2011)

2) G. Katata, H. Terada, H. Nagai, and M. Chino: J. Environ. Radioactiv., 111, 2-12 (2012).

3) G. Katata, M. Ota, H. Terada, M. Chino, and H. Nagai: J. Environ. Radioactiv., 109, 103-113 (2012).

4) H. Terada, G. Katata, M. Chino, and H. Nagai: J. Environ. Radioactiv., 112, 141-154 (2012).

5) H. Terada, H. Nagai, A. Furuno, T. Kakefuda, T. Harayama, and M. Chino: Trans Atomic Energy Soc. Jpn. [in Japanese with English abstract], 7, 257-267 (2008).

6) S. Hirao, H. Yamazawa, and T. Nagae: J. Nucl. Sci. Technol., 50, 139-147 (2013). 